Schramm, M. \& Hestrin, S. (1954). J. gen. Microbiol. 11, 123-129.

\title{
Factors affecting Production of Cellulose at the Air/ Liquid Interface of a Culture of Acetobacter xylinum
}

\author{
BY M. SCHRAMM AND S. HESTRIN \\ Laboratory of Microbiological Chemistry, Department of Biochemistry, \\ Hebrew University-Hadassah Medical School, Jerusalem, Israel
}

SUMMARY: The cells in a developing culture of Acetobacter xylinum in a static liquid glucose medium were swept to the surface by the flotation of a submerged cellulose net. The net was probably pulled to the surface by adsorbed carbon dioxide given off by the metabolizing cells. The relatively high oxygen pressure at the aerated surface of a static liquid culture mediated the rapid production of cellulose in the surface layer. $A$. xylinum produced less cellulose in swirled than in static nutrient medium. Cellulose in the swirled medium occurred in the form of remarkable bodies, spherical, jointed or stellate in form, up to $10 \mathrm{~mm}$. in size. The swirling favoured the overgrowth of the wild type organism by mutants which were specifically deficient in the ability to form cellulose. On the basis of the type of colonial growth and of the pellicle characteristics, two groups of $A$. xylinum mutants with an extreme deficiency in cellulose-forming ability and one with an intermediate degree of deficiency were differentiated.

An extracellular framework consisting of cellulose characterizes the growth habitat of Acetobacter xylinum (Franz \& Schiebold, 1943; Aschner \& Hestrin, 1946; Frey-Wyssling \& Muhlethaler, 1946). The understanding of the morphogenesis of this remarkable structure is relevant to efficient technical use of $\boldsymbol{A}$. xylinum as an oxidizing organism and as a cellulose source (Evans \& Hibbert, 1947) and to the use of the organism as a model system in which physical and chemical aspects of cell-wall production can be examined outside the cell (Hestrin, Aschner \& Mager, 1947; Muhlethaler, 1948, 1949; Hestrin, 1953; Hestrin \& Schramm, 1954). In the present communication the mechanics of the flotation and thickening of a xylinum pellicle are considered and the effects of agitation on the expression of the cellulose-synthesizing activity and its heritable transmission are described.

\section{METHODS}

Observations on growth of the pellicle under conditions of static culture were conducted in glass cylinders. Agitation of cultures was effected by swirling them in conical Erlenmeyer flasks with the aid of a Ross-Kershaw shaking apparatus maintained in a thermostat. The medium was inoculated with one-twentieth of its volume of the liquid phase of a starter culture. Loose cotton-wool stoppers which did not unduly obstruct oxygen supply were employed. The temperature in all experiments was $30^{\circ}$.

The liquid medium was of the following composition $(\%, w / v)$ : glucose $2 \cdot 0$, Bactopeptone (Difco) 0.5; yeast extract (Difco) 0.5 ; initial $\mathrm{pH}$ value 6. A solid medium contained also $2 \cdot 5 \%$ of agar.

To determine the total fresh weight of a pellicle, it was lifted from the surface of the culture, hung freely on a glass peg for $15 \mathrm{~min}$. to drain away the excess 
water, and weighed. The pellicle was then gently stirred with a large volume of distilled water for $90 \mathrm{~min}$. to remove soluble material, and again weighed after drying at $80^{\circ}$ over $\mathrm{P}_{2} \mathrm{O}_{5}$ in vacuo. Cellulose was determined by the method previously described (Schramm \& Hestrin, 1954).

\section{RESULTS}

\section{The flotation of the pellicle}

An inoculum of Acetobacter xylinum added to liquid medium formed a diaphanous submerged net (Muhlethaler, 1948; Kaushal, Walker \& Drummond, 1951). The culture fluid on the 1st day after inoculation, and before the formation of a visible surface pellicle, was somewhat turbid. Under static conditions in a shallow-layer liquid culture, a visible pellicle was present on the surface of the liquid after 2 days. The medium had cleared at this stage, the diaphanous net having risen to the surface with most of the bacteria enmeshed in it. The clearing of the medium at the same time as the appearance of the surface film suggested that a special mechanism had swept the cells into the surface layer. It was shown that the xylinum cells when acting on glucose mediate a slow evolution of carbon dioxide. A washed suspension of the cells shaken under oxygen at $30^{\circ}$ in $4 \%$ glucose solution in $0.02 \mathrm{M}$-citrate ( $\mathrm{pH} 6.4$ ) formed carbon dioxide at a sustained rate of approximately $0.44 \mu \mathrm{mole} / \mathrm{mg}$. cells (dry weight)/hr. as determined manometrically by Warburg's indirect method. Any gas evolved by a growing culture in an undisturbed liquid nutrient medium would accumulate on the surfaces of the cellulose fibrils; sometimes macroscopic bubbles of gas were detected in the submerged mesh. The gas probably caused the flotation of the net, and the net presumably pulled the bacteria to the surface.

\section{The thickening of the pellicle}

Synthesis of cellulose from glucose by a suspension of non-proliferating bacteria is a function of oxygen supply (Hestrin et al. 1947). The rate of cellulose production was negligible under nitrogen, about one-half optimal under air and approached closely the optimum rate under $100 \%$ oxygen in a reciprocally shaken suspension of cells in glucose solution. A relatively rapid increase of cellulose might thus be expected to take place as a result of the concentration of cells of a growing culture in the aerated surface zone. In a growing culture, moreover, the oxygen supply in the surface layer would mediate increase of the mass of the cells and of cellulose-forming enzyme and thus further augment cellulose production. Within certain limits, moreover, the system would be a self-accelerating one, since the increase of the cellulose content of the incipient surface film would serve to facilitate the retention of the cells in the surface.

The yields of cellulose found after 2- and 5-day periods of growth under air in static culture are illustrated by the data shown in Table 1 . The yields of cellulose from the static cultures demonstrate the time lag preceding the colonization of the liquid surface and the dominant role of the area of the surface exposed to the gas phase in conditions of static culture. In agitated 
cultures, on the other hand, the total volume of the medium was the factor which determined the amount of cellulose obtained.

The observations just summarized confirm the view that the localization of cellulose production at the surface of the static liquid culture was determined by the oxygen pressure. It need not be assumed that mechanical and electrical vector fields in the surface played a causal role in the polymerization, although these might play a part in the secondary extracellular aggregation and crystallization of the cellulose molecules (cf. Franz \& Schiebold, 1943; Aschner \& Hestrin, 1946; Frey-Wyssling \& Muhlethaler, 1946; Muhlethaler, 1948, 1949; Kaushal et al. 1951; Ranby, 1952a, b).

Table 1. Influence of growth time, volume of medium and agitation on the production of cellulose by $\mathbf{A}$. xylinum growing on liquid glucose medium

The glucose medium was inoculated with $1 / 150$ th of its volume of fluid culture and distributed into conical flasks $(50 \mathrm{ml}$.). Technique of cultivation and analytical procedure were as described in the section 'Methods'.

\begin{tabular}{|c|c|c|c|c|c|c|c|}
\hline \multirow{2}{*}{$\begin{array}{c}\text { Period } \\
\text { of } \\
\text { incubation } \\
\text { (days) }\end{array}$} & \multirow{2}{*}{$\begin{array}{c}\text { Volume } \\
\text { of } \\
\text { medium } \\
\text { (ml.) }\end{array}$} & \multirow{2}{*}{$\begin{array}{c}\text { Conditions } \\
\text { of } \\
\text { growth }\end{array}$} & \multicolumn{2}{|c|}{$\begin{array}{c}\text { Total wt. of pellicle } \\
\text { (mg.) }\end{array}$} & \multirow{2}{*}{$\begin{array}{l}\text { Yield of } \\
\text { cellulose } \\
\text { (mg.) } \\
\text { (b) }\end{array}$} & \multirow{2}{*}{$\begin{array}{c}\text { Non- } \\
\text { cellulose } \\
\text { dry weight of } \\
\text { pellicle (mg.) } \\
(c=a-b)\end{array}$} & \multirow{2}{*}{$\begin{array}{l}\text { Non- } \\
\text { cellulose: } \\
\text { cellulose } \\
\text { ratio } \\
(c / b)\end{array}$} \\
\hline & & & Wet & $\begin{array}{c}\text { Dry } \\
(a)\end{array}$ & & & \\
\hline \multirow[t]{4}{*}{$\mathbf{2}$} & $\mathbf{3 0}$ & Static & 419 & - & $2 \cdot 10$ & - & 一 \\
\hline & 15 & Static & 432 & 5 & $1 \cdot 78$ & $3 \cdot 2$ & 1.8 \\
\hline & 30 & Swirled & - & - & 0.84 & - & - \\
\hline & 15 & Swirled & - & - & $0 \cdot 45$ & - & - \\
\hline \multirow[t]{4}{*}{5} & 30 & Static & 2680 & 34 & $13 \cdot 2$ & $20 \cdot 8$ & $1 \cdot 6$ \\
\hline & 15 & Static & 2673 & 32 & $11 \cdot 7$ & $20 \cdot 3$ & $1 \cdot 7$ \\
\hline & 30 & Swirled & - & - & $4 \cdot 80$ & - & - \\
\hline & 15 & Swirled & - & - & $2 \cdot 60$ & - & - \\
\hline
\end{tabular}

Cellulose production being thus a function of the oxygen pressure, it was expected that the increase of the mass of cellulose in a pellicle would occur principally in the upper part of it. The underpart of the pellicle would be pushed by this process progressively downwards into zones of decreasing oxygen pressure and lesser activity. This was experimentally verified as follows. Particles of cork were added to a xylinum culture on the same day as the inoculum. The particles floated on the surface and were incorporated within the forming pellicle. When the pellicle later increased in thickness, the cork particles remained fixed within the relatively transparent underpart of the pellicle, thus showing unambiguously that the accretion of cellulose had occurred principally in the upper part of the pellicle.

Whereas the upper part of the mature pellicle was generally opaque and quite firm, the under part was relatively transparent and soft, and frequently supported a fragile transparent sac which hung loosely from the undersurface. Kaushal et al. (1951) suggested that the relative transparency and lack of rigidity of the underpart of a pellicle might be indicative of a greater proportion of short and imperfectly oriented nascent chains of cellulose. However, when the cells of an opaque and firm pellicle are dissolved by storage in dilute 
sodium hydroxide solution, the recovered cellulose gel is highly transparent and soft. Yet the appearance of this purified product under the electron microscope, its chemical properties, $\mathbf{X}$-ray diffraction diagram and infra-red absorption spectrum, are all those of a high-polymer crystalline cellulose (for example, see Ranby, $1952 a$; Forziati \& Rowen, 1951).

The underpart of the pellicle has been shown above to be the oldest part. Thus the presence in this part of a higher proportion of short cellulose chains or of less well oriented chains appears to be rather unlikely, and some other explanation for the special properties displayed by this part may be desirable. It should not be overlooked that while the cellulosic extracellular gel component of the pellicle contributes the bulk of the gel volume, the dry weight of the pellicle is largely due to the bacteria in it (Table 1). It is therefore apparent that physical properties of a pellicle such as its opacity, rigidity, tensile strength and specific gravity could be functions of the condition and relative proportion of bacteria of the pellicle, and need not depend only on the nature, concentration and disposition of the cellulose chains in the extracellular gel fraction.

\section{Influence of agitation on the production of cellulose}

Since the rapid production of cellulose on the surface of a static liquid culture was determined by the relatively high oxygen pressure at the surface, it was anticipated that cellulose production in an agitated aerated liquid would take place throughout the volume of the medium. In fact, when the Acetobacter xylinum was transferred from static liquid culture to a swirled medium, it grew and synthesized cellulose. However, the pattern of the growth of cells and of the production of cellulose in the swirled medium showed important departures from the pattern in static liquid cultures. (a) The cultures in the swirled medium formed much less cellulose (Table 1). (b) The cellulose in the swirled cultures occurred in the form of remarkable macroscopic bodies (Pl. 1, fig. 1) in size up to $c .10 \mathrm{~mm}$. diam. and spherical, jointed or stellate in form, resembling in appearance but exceeding in size the bodies of cellulose which are also formed by freeze-dried $\boldsymbol{A}$. xylinum when it is swirled in buffered glucose solution. (c) Both at first transfer when the growth was allowed to continue for more than three days, and in swirled subcultures, the cells growing in swirled medium gradually lost ability to form cellulose and assumed a diffuse growth habit. To minimize the possibility that the late diffuse growth had been caused by contamination, the stock was purified by repeated plating on glucose agar. Four single-colony isolates from 4-day agar cultures all showed normal pellicle-forming ability in static liquid medium. In swirled cultures they showed the same behaviour as the original stock. The clones were transferred in swirled medium serially at intervals of $72 \mathrm{hr}$. With increase in the number of transfers, the growth in every case became more diffuse and there was a gradual diminution and finally disappearance of cellulose production (Pl. 1, fig. 2). The same observation was also made when the experiment was repeated with a strain of $A$. xylinum which had been placed at our disposal by Prof. M. Aschner. 


\section{Isolation and classification of mutants deficient in cellulose-forming ability}

In a static liquid medium a floating pellicle insures an abundant oxygen supply to the cells. In a swirled liquid, on the other hand, the cells need not be floated by the pellicle in order to obtain oxygen and actively proliferating forms which were deficient in cellulose-forming ability might thus gain ascendancy. The view that the decline of cellulose production in swirl-culture is associated with overgrowth of the culture by cellulose deficient mutants was supported as follows.

A swirl-culture of fourth transfer, derived from a single-colony isolate of the Acetobacter xylinum stock, was plated on agar, and the properties of the isolated clones studied. On the basis of the types of colonies grown on agar (Pl. 2, fig. 3) and of the properties of the pellicles formed by the cultures on static liquid glucose medium (Table 2), the cultures were classified as wild-type or

\section{Table 2. Characteristics of surface pellicle of wild-type and cellulose-deficient mutants of A. xylinum}

Cultures were grown in static liquid glucose medium in test tubes and characterized on the 7 th day.

\begin{tabular}{|c|c|c|c|}
\hline \multirow[b]{2}{*}{ Organism } & \multicolumn{3}{|c|}{ Properties of pellicle } \\
\hline & $\begin{array}{l}\text { Thickness } \\
\text { (mm.) }\end{array}$ & $\begin{array}{c}\text { Coherence in } \\
4 \% \mathrm{NaOH}\end{array}$ & $\begin{array}{l}\text { Coherence when } \\
\text { agitated gently }\end{array}$ \\
\hline Wild-type & 5 & Unchanged & Unchanged \\
\hline Intermediate & $1^{*}$ & Unchanged & Unchanged \\
\hline Celluloseless I & 1 & Dispersed & Flakes \\
\hline Celluloseless II & 1 & Dispersed & Dispersed \\
\hline
\end{tabular}

mutant. The mutant cultures were subclassified into three groups, two of them (celluloseless I and II) with extreme or absolute deficiency in cellulose-forming ability, and one with an intermediate degree of deficiency. All these mutants displayed characteristic Acetobacter properties, including growth in the form of rods of varying length, capacity to catalyse restricted oxidations with formation of acetic acid from ethanol and of acids from glucose, and formation of a zoogleal film on liquid medium. Since the zoogleal films which are formed by the 'celluloseless' mutants on the surface of liquid nutrient medium are fragile and dissolved by hot $4 \%$ sodium hydroxide, the cementing substance of these films is probably a substance other than cellulose.

The good growth rates of the mutants and their oxidative activities support the view that the states of deficiency of the mutants represent a specific lesion in the apparatus of cellulose synthesis rather than a generalized loss of metabolic vigour. The degree of the cellulose deficiency of the cultures was stably maintained in static liquid medium through ten serial transfers. When the wild-type was grown in mixed culture with any one of the mutants on a static liquid medium from a mixed inoculum containing a large preponderance of the mutant $(0.05 \mathrm{ml}$. wild-type culture $+2.0 \mathrm{ml}$. mutant culture as mixed 
inoculum for $10 \mathrm{ml}$. medium), the wild-type rapidly gained the ascendancy. After a few transfers in static growth conditions, the mixed culture displayed the phenotypic characteristics of the wild-type, namely a cellulosic surface pellicle with clear underlying fluid. Therefore, the observed absence of a cellulose pellicle in the pure cultures of the mutants can be interpreted as affording evidence that genetic reversion to the wild-type is relatively rare. The observations are in agreement with the conclusion that the decline of the ability of Acetobacter xylinum to produce cellulose can occur by way of mutation in one or more steps. In view of the potential usefulness of the xylinum mutants as biochemical tools, it will be of particular interest to identify the enzyme lesion in the mutants more definitely and to ascertain whether the xylinum mutant strains accumulate a cellulose precursor.

The authors are grateful to Prof. M. Aschner for kind advice. Part of the work describing the isolation of mutants was carried out by one of us (M.S.) in work leading to the degree of Ph.D.

\section{REFERENCES}

Aschner, M. \& Hestrin, S. (1946). Fibrillar structure of cellulose of bacterial and animal origin. Nature, Lond. 157, 659.

Evans, T. H. \& Hibbert, H. (1947). Bacterial polysaccharides. Rep. Sug. Res. $F d n, N . Y .6$.

Fonziati, F. H. \& Rowen, J. W. (1951). Effect of changes in crystalline structure on the infrared absorption spectrum of cellulose. J. Res. nat. Bur. Stand. 46, 38-42.

Franz, E. \& Schiebold, E. (1943). Beiträge zur Struktur der Bakterienzellulose. Makromol. Chem. $1,3$.

Frey-Wyssling, A. \& Mühlethaler, K. (1946). Submicroscopic structure of cellulose gels. J. Polym. Sci. 3, 172.

Hestrin, S. (1953). Mechanism of degradation and synthesis and some biological activities of intercellular bacterial polysaccharides. Symp. Microbiol. Metabolism. Proc. VIth Congr. Int. Microbiol., Rome, p. 63.

Hestrin, S. \& Schramm, M. (1954). Preparation of freeze dried cells capable of polymerizing glucose to cellulose. Biochem. $J$. (in the Press).

Hestrin, S., Aschner, M. \& Mager, J. (1947). Synthesis of cellulose by resting cells of Acetobacter xylinum. Nature, Lond. 159, 64.

Kaushal, R., Walker, T. K. \& Drummond, D. G. (1951). Observations on the formation and structure of bacterial cellulose. Biochem. J. 50, 128.

MühlethaleR, K. (1948). Elektronenoptische Untersuchungen über den Feinbau von Gelen. Makromol. Chem. $2,143$.

MühlethaleR, K. (1949). The structure of bacterial cellulose. Biochim. Biophys. Acta, 3, 527.

Ranby, B. G. (1952a). Physico-chemical investigations on bacterial cellulose. Ark. Kemi, 4, 249.

Ranby, B. G. (1952b). The cellulose micelles. Tappi, 35, 53.

Schramm, M. \& Hestrin, S. (1954). Synthesis of cellulose by Acetobacter xylinum. I. Micromethod for determination of celluloses. Biochem. J. 56, 163. 
Journal of General Microbiology, Vol. 11, No. 1

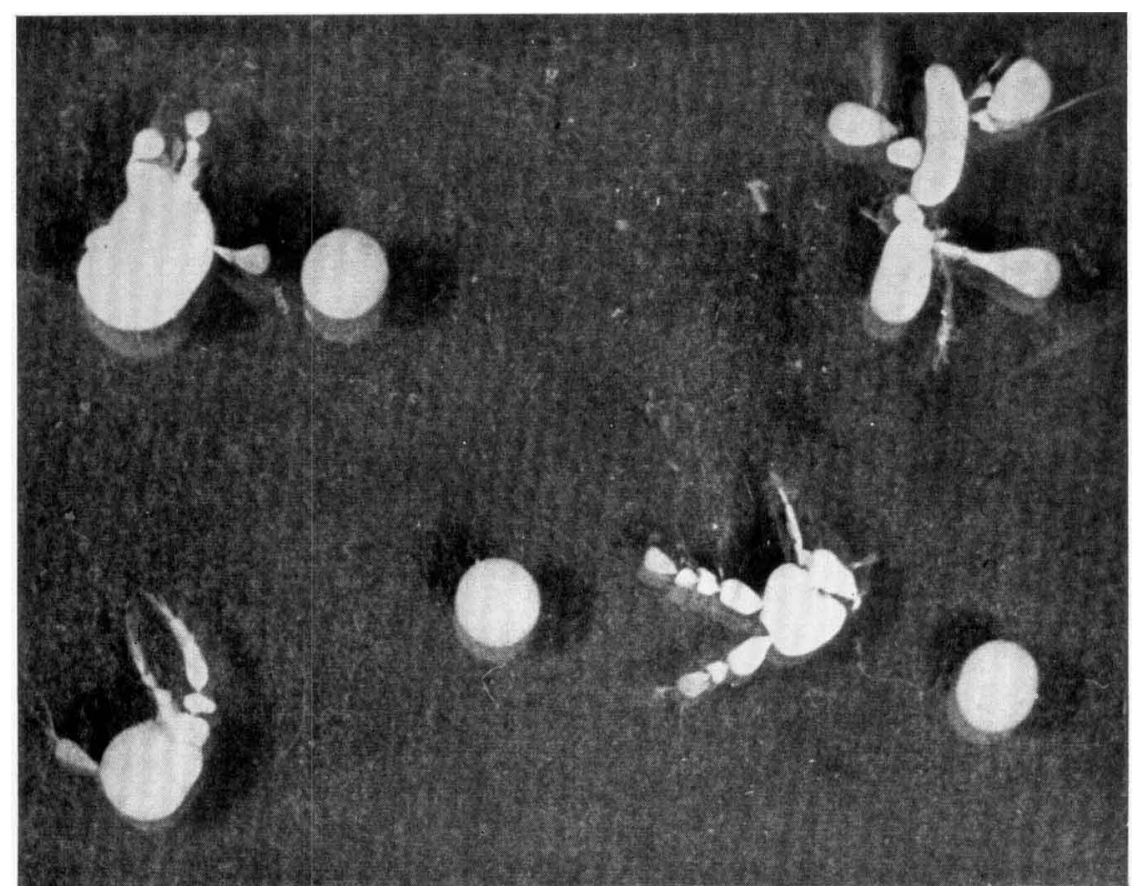

Fig. 1

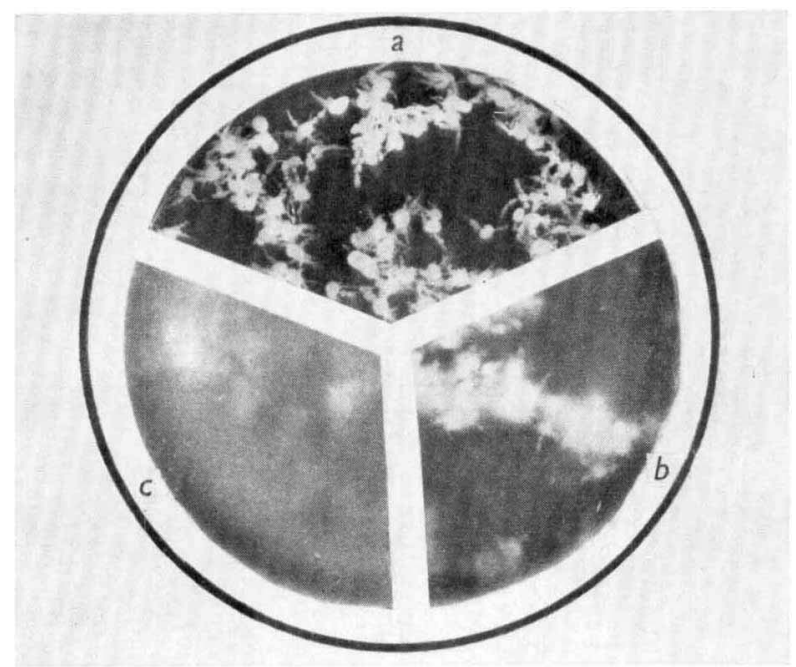

Fin. 2

M. Schramm \& S. Hestrin-Production of clelulose by A. xylingu. Plate 1 
Journal of General Microbiology, Vol. 11, No. 1

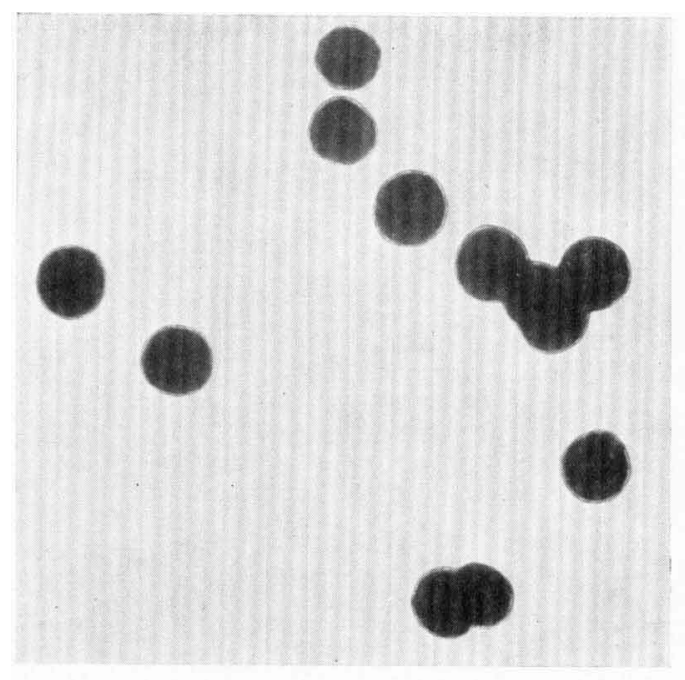

Quadrant 1

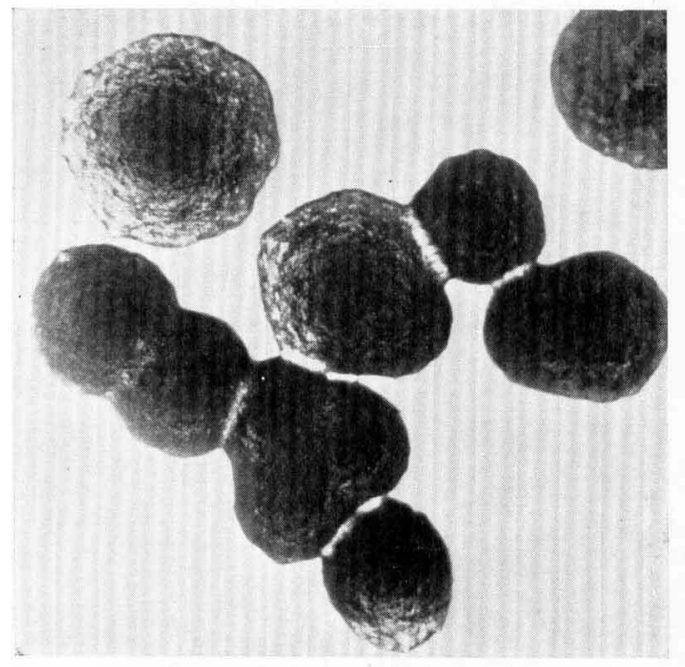

Quadrant 3

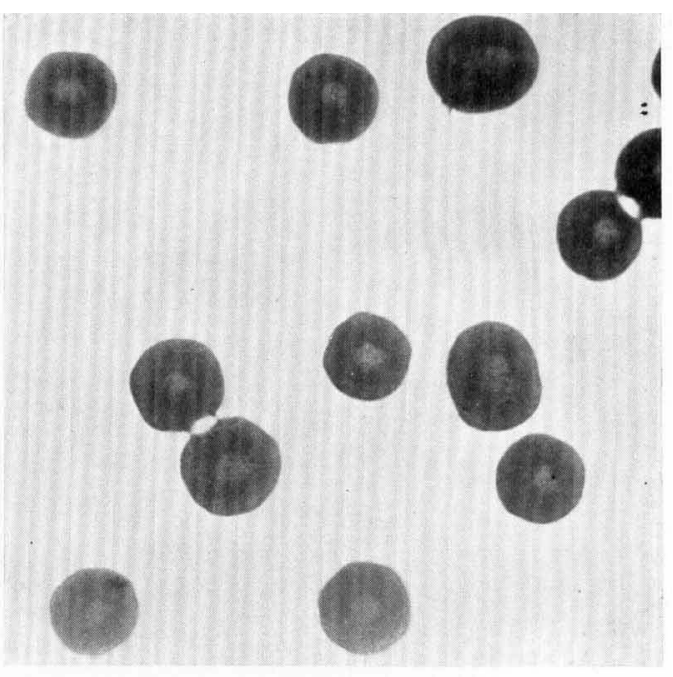

Quadrant 2

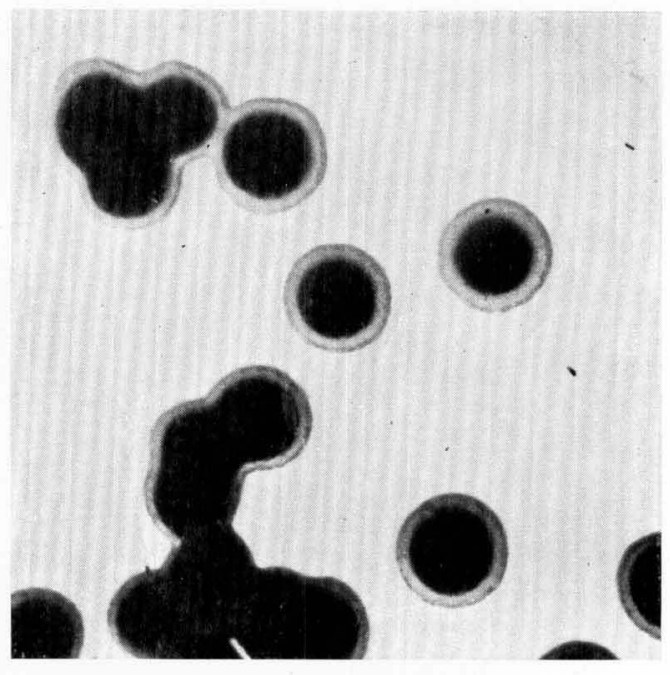

Quadrant 4

M. Schramm \& S. Hestrin-Production of cellulose by A. xylinum. Plate 2 


\section{EXPLANATION OF PLATES}

Plate 1

Fig. 1. Spherical and jointed bodies formed by Acetobacter xylinum on transfer from static to swirled growth medium. $\times 4 \cdot 5$.

Fig. 2. Gross appearance of a swirl-culture of $A$. xylinum. Sectors $a, b$ and $c$, are typical fields from photographs of a swirl-culture of the 1st, 2nd and 3rd transfer respectively. $\times 1$.

\section{Plate 2}

Fig. 3. Colonies of $A$. xylinum wild-type and of mutants deficient in the ability to produce cellulose. The colonies on glucose agar were photographed, after 4 days of growth, as observed under the microscope by transmitted light. $\times 22$. For characteristics of pellicles formed on liquid glucose medium see Table 2. Quadrant 1: colonies of wildtype; characteristically dwarf and heavily opaque. Quadrant 2: colonies of a mutant with intermediate deficiency in cellulose-forming ability; showing less opacity than the wild-type and a relatively bright central zone. Quadrant 3: colonies of the mutant designated celluloseless I; characteristically rough. Quadrant 4: colonies of mutant designated celluloseless II; with characteristic translucent outer rim.

(Received 25 February 1954) 